\title{
Corela
}

Cognition, représentation, langage

HS-8 | 2010

L'interpellation

\section{L'interpellation comme signe évaluateur des intentions et de l'animation de la parole dans les interactions orales transformées sur le terrain de l'enseignement/apprentissage du F.L.E.}

\section{Alain Brouté}

\section{(2) OpenEdition}

\section{Journals}

Édition électronique

URL : http://journals.openedition.org/corela/1760

DOI : $10.4000 /$ corela. 1760

ISSN : 1638-573X

Éditeur

Cercle linguistique du Centre et de l'Ouest - CerLICO

Référence électronique

Alain Brouté, «L'interpellation comme signe évaluateur des intentions et de l'animation de la parole dans les interactions orales transformées sur le terrain de l'enseignement/apprentissage du F.L.E. », Corela [En ligne], HS-8 | 2010, mis en ligne le 16 novembre 2010, consulté le 30 avril 2019. URL : http:// journals.openedition.org/corela/1760 ; DOI : 10.4000/corela.1760

Ce document a été généré automatiquement le 30 avril 2019

\section{(†) (2)}

Corela - cognition, représentation, langage est mis à disposition selon les termes de la licence Creative Commons Attribution - Pas d'Utilisation Commerciale - Partage dans les Mêmes Conditions 4.0 International. 


\title{
L'interpellation comme signe évaluateur des intentions et de l'animation de la parole dans les interactions orales transformées sur le terrain de l'enseignement/ apprentissage du F.L.E.
}

\author{
Alain Brouté
}

Si «pour Le Robert \& CLE international (2007, 545) interpeller, c'est adresser brusquement la parole à quelqu'un » ou « apostropher », « questionner quelqu'un sur son identité » ou encore " provoquer un intérêt chez quelqu'un », et " pour le Dictionnaire de l'Académie Française (1932-1935) c'est s'adresser à quelqu'un à la volée, de façon soudaine, brusque, pour le questionner ou l'insulter " ou encore "pour lui demander quelque chose ", alors nous avons bien affaire à une action propre de la communication interactionnelle interpersonnelle, la communication interactionnelle en question pouvant être assimilée au domaine des interactions orales, de face à face ou autres, et de leur interlocution improvisée ou plus ou moins spontanée. En considérant l'interpellation comme un objet générique, structurant et, peut-être, déterminant de l'interaction orale, je me suis donc intéressé particulièrement à la relation entre des indices de cette interpellation, qui sont inscrits dans l'improvisation de l'interlocution, et leurs effets sur les interactions orales. Pour cela, j'ai privilégié l'observation du terrain de l'enseignement/apprentissage du F.L.E. (Français Langue Etrangère) et, plus particulièrement, les interactions orales développées en classe dans cette langue qui correspondent aujourd'hui à la cinquième compétence du Cadre Européen Commun de Référence. C'est ainsi que j'ai pu observer plusieurs types d'interpellations lors d'interactions orales transformées ou jeux de rôles, plus ou moins préparés ou improvisés, pratiqués par des apprenants de plusieurs âges et niveaux. Le fait de choisir 
un corpus d'interactions orales transformées est d'autant plus pertinent pour notre analyse que l'activité qui consiste à transformer, donc à simuler une activité modèle, nous ramène à « un travail de différenciation suggérant que nous puissions nous distancier de nous au sens où le rapport de soi à soi crée la possibilité du lien social (Brouté, 2006, 22)». C'est ainsi que la simulation d'une séquence d'activité ou transformation de cette activité est « pour E. Goffman $(1991,489)$ la garantie par excellence d'une bonne analyse des cadres ", d'autant que " les relations entre la conversation et le monde connaissent des relâchements constants et de tels relâchements la rendent plus vulnérable que d'autres activités aux transformations (Brouté, 2006, 40) ». En fait, dans le prolongement de l'analyse d'une mise en scène de la vie quotidienne proposée par E. Goffman (1973, b), nous pouvons admettre que ceux qui s'engagent dans une interaction orale, qui soit de face à face ou autre, s'impliquent en même temps dans un rôle, supposant en cela une transformation continue de l'interaction en question. Par ailleurs, dans les interactions orales, et a fortiori en langue étrangère, le plus difficile consiste « comme nous le suggère J-F. de Raymond $(1980,98)$, en la simultanéité entre l'action du sujet et la modification de la situation qui lui est imposée par celle du partenaire, créant ainsi par l'adaptation de la stratégie à la disposition et au mouvement de l'autre, une situation de changement permanent où la seule façon de rester contemporain de ce devenir, et de le conduire, est bien d'improviser ». Alors, les interactions orales transformées et leurs interpellations à observer doivent être de l'ordre de l'improvisation ou, pour le moins, tendre vers ce caractère improvisé. Aussi, je vous présenterai dans un premier temps un échantillon d'indices catégorisés de ces interpellations puis, dans une seconde partie, j'exposerai quelques éléments méthodologiques (activités, jeux de rôles, ateliers, évaluations...) qui permettent d'utiliser les observations précédemment mentionnées dans le but de favoriser le développement d'une pratique et d'une compétence d'interaction orale en F.L.E. et, éventuellement, pour une langue seconde ou une langue maternelle.

\section{Les indices d'interpellation dans le cadre d'interactions orales transformées ou jeux de rôles en classe de F.L.E.}

2 En tant qu'action inscrite dans l'improvisation de l'interlocution et telle que définie par les différents dictionnaires, dont les deux précités au début du texte, l'interpellation marque une volonté d'interrompre le cours des événements et d'en changer la progression en essayant de provoquer ou une interaction avec l'interpelé ou une modification intentionnelle du flux de l'interaction en cours avec cet interpelé. De cette sorte, l'interpellation est susceptible de se baser sur un ou plusieurs signes ayant une fonction non seulement d'indicateur de gestion de la parole, traduisant une intention quant à cette parole avant ou pendant l'interaction orale, mais également une fonction de marqueur d'animation de cette interaction, venant se surajouter au signe de gestion de la parole et permettant notamment de commenter l'action future ou en cours sous la forme d'une évaluation cognitive ou d'une évaluation empathique marquant fondamentalement le style de l'interpellant dans l'interaction. Pour ce qui est de l'analyse en tant que signe de gestion de la parole, nous savons " grâce aux travaux de H. Sacks, E.H. Schegloff et G. Jefferson (1974), puis « ceux de S. Duncan et D.W. Fiske (1977)» que cette gestion de la parole ou des tours de parole dans l'interaction se développe à travers toute une gamme de signaux phatiques de la part du locuteur, lui permettant de maintenir la parole ou de 
la céder, et de signaux régulateurs de la part de l'interlocuteur, lui permettant d'être candidat à la parole ou de la laisser momentanément au locuteur. C'est ainsi que l'interpellation peut se produire non seulement au tout début de l'interaction orale de face à face ou autre, et nous parlerons alors d'interpellation d'intervention, mais également au cours de cette interaction orale afin d'interpeller autrui pour lui demander de prendre la parole. Nous aurions affaire en l'occurrence seulement à 2 des 4 mouvements de la gestion: la cession et la candidature. Cette interpellation pouvant concerner tous les participants à l'interaction, nous parlerons alors d'interpellation de désignation pour le locuteur invitant l'autre à prendre la parole et d'interpellation d'interposition pour l'interlocuteur posant sa candidature à cette parole.

Pour ce qui est de l'analyse en tant que marqueur d'animation, l'interpellation illustre un style propre d'intervention, de désignation ou d'interposition de la part de l'interpellant, style qui interpelle autrui simultanément à travers les indicateurs de gestion de la parole, le tout reposant sur des termes d'adresse illustrés par des marqueurs verbaux, acoustiques et kinésiques sous la forme d'illustratifs, de quasi-linguistiques et d'extracommunicatifs que nous allons détailler plus avant. Nous avons donc deux niveaux d'analyse, en l'occurrence une analyse plus technique concernant les intentions par rapport à la parole en interaction qui nous renseigne sur des degrés premiers de participation ou d'engagement des interlocuteurs apprenants en interaction et une analyse plus qualitative permettant d'étudier les degrés de coopération cognitive et empathique des mêmes interlocuteurs apprenants, l'ensemble nous informant sur les difficultés rencontrées et nous suggérant d'éventuelles actions pour y remédier.

\subsection{Analyse de l'interpellation comme signe de gestion de la parole en interaction}

\subsubsection{La prise de parole ou interpellation pour initier l'interaction orale de face à face ou autre}

Cette prise de parole ou «appel à l'interaction » illustre une intention significative plus ou moins forte d'intervenir pour rompre une absence de communication entre les êtres ou pour intervenir dans une interaction en cours entres d'autres personnes. L'interpellant intervenant qui sollicite l'interaction s'adresse alors à un ou plusieurs interlocuteurs potentiels qui peuvent percevoir ou pas l'intention et sa manifestation sémiotique et qui peuvent ou pas accepter l'intervention et sa conséquence. Dans le cas de l'intervention, l'interpellation est le plus souvent illustrée par des appellatifs sous la forme de :

- Onomatopées verbales, vocales et/ou kinésiques : «Eh », « Euh », raclements de gorge...

Marqueurs verbaux d'appel : « Dis donc ! », « Au fait... ! », « Attends... ! »,...

Rituels de contact verbaux, gestuels et acoustiques d'ordre phatique.

Déictiques pour attirer l'attention de l'interlocuteur potentiel.

9 Marqueurs kinésiques d'appel : Regard brusque en direction de l'interlocuteur potentiel, regard soutenu en direction de ce possible interlocuteur, ces regards pouvant être accompagnés d'un mouvement de tête, d'une mimique expressive, d'une gestuelle appellative (cf. photo ci-dessous). 


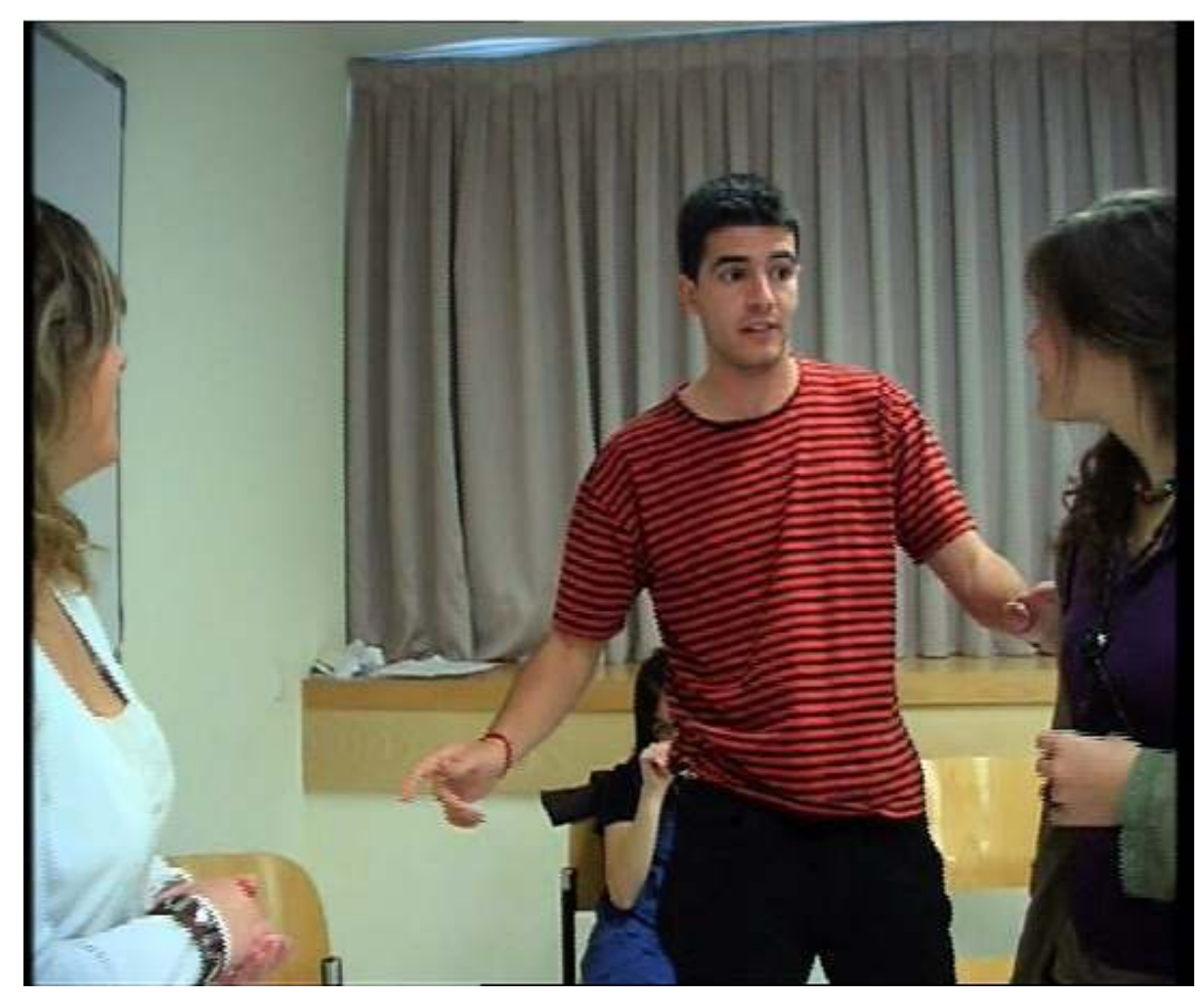

10 Tel que nous l'avons suggéré auparavant, les interactions orales transformées de l'ordre $\mathrm{du}$ comme si sur le terrain de l'enseignement/apprentissage du F.L.E. ne peuvent connaître des interpellations d'intervention que si les participants font preuve d'un minimum de spontanéité, en l'occurrence lors de jeux de rôles suffisamment improvisés, comme dans le cas illustré par la photo antérieure au cours duquel intervient spontanément un apprenant extérieur à l'interaction orale en cours sans que les participantes à cette interaction aient été averties. Si elles avaient été informées avant de cette interpellation, nous parlerions alors plutôt d'une interpellation d'interposition dans la mesure où nous considérons que le fait de reconnaître a priori un participant comme un interlocuteur potentiel ne peut entraîner que la modification plus ou moins accordée du flux du discours et non celle de la nature même de ce discours comme lors d'une interpellation d'intervention. Dans l'exemple du jeu de rôle improvisé illustré, l'interpellant est intervenu sans prévenir les deux interlocutrices, interrompant ainsi non seulement le flux de leur interaction, mais également leur discours en créant une nouvelle situation et un nouveau thème de discours. Au cas où ils se seraient mis d'accord auparavant sur l'interpellation en question, le caractère d'intervention aurait perdu sa raison d'être et nous aurions affaire à une interpellation propre d'un interlocuteur muet jusque lors et s'interposant dans le discours en interaction pour revendiquer à son tour la parole. Cet exemple nous permet de voir la différence essentielle existant entre les deux types d'interpellations en interaction. En effet, nous aurions d'un côté celles illustrant une intervention de l'interpellant et qui modifient profondément la situation en provoquant une interaction nouvelle et, de l'autre, celles qui ne font que modifier le cours du discours de l'interaction existante par le biais d'une désignation ou d'une interposition 


\subsubsection{L'interpellation en cours d'interactions orales de face à face ou autres}

11 Comme nous l'avons avancé en introduction de cette première partie du texte, l'interpellation peut être illustrée par des signes de gestion de la parole en interaction. Toutefois, si les signes de la gestion de la parole en interaction sont de 4 types, il semble que la moitié seulement de ces signes sont susceptibles de représenter une interpellation. En effet, lors d'interactions orales, de face à face ou autres, les interlocuteurs peuvent recourir à 4 grands types de signes pour gérer la parole selon qu'ils sont en possession ou pas de cette parole :

12 -Des signes de maintien de la parole de la part du locuteur

-Des signes de passation de la parole de la part du locuteur

-Des signes de candidature à la parole de la part de l'interlocuteur

-Des signes d'invitation à garder la parole de la part de l'interlocuteur

13 Si l'interpellation marque une interruption du cours des événements, mais n'est pas de l'ordre de l'intervention, elle ne peut illustrer alors qu'une interruption dans le flux continu de l'interaction orale. Il nous faut interroger chaque type de signes de gestion de la parole afin de s'assurer qu'ils peuvent représenter une interpellation. Dans le cas des signes concernant le locuteur, ceux du maintien correspondent à une volonté de poursuivre la locution et ne peuvent se manifester que si le locuteur a une bonne raison de penser que son interlocuteur va lui quitter cette parole. Or, cela suppose que ce dernier a manifesté auparavant une candidature à la parole. Il ne s'agit donc pas d'une interpellation, mais plutôt d'une réaction à une interpellation de l'interlocuteur qui souhaite prendre la parole détenue par le locuteur. Dans le cas de la passation de la parole, cette dernière est susceptible de correspondre à une réaction à une candidature de l'interlocuteur, mais elle peut aussi illustrer une interruption du flux de la conversation dans la mesure où le locuteur s'adresse à l'interlocuteur pour que celui-ci prenne possession de la parole sans l'avoir demandée auparavant.

Dans le cas des signes concernant le ou les interlocuteurs, si les signes de candidature illustrent une volonté de changer le cours du flux de la parole comme nous l'avons montré auparavant et peuvent être assimilés à un type d'interpellation, pour ce qui est des signes d'invitation à garder la parole adressés au locuteur, il semble qu'il ne peut pas s'agir d'une interpellation dans la mesure où cette invitation ne rompt en rien le cours des événements puisque le locuteur va poursuivre son discours au moins pour quelques instants, ou il s'agit d'une réponse à une interpellation pour passer la parole de la part de l'interlocuteur. Aussi, nous voyons comment seuls les signes de gestion de la parole en interaction pour passer cette parole de la part du locuteur ou poser sa candidature de la part de l'interlocuteur sont susceptibles de représenter une interpellation au cours de l'interaction orale, de face à face ou autre. Nous observons ainsi comment les signes de la gestion de la parole qui correspondent à une interpellation illustrent une rupture dans le flux conversationnel dans la mesure où l'individu manifeste une intention de modifier ce flux et son rôle jusque lors dans l'interaction orale pour passer de locuteur à interlocuteur ou réciproquement.

Pour que le locuteur ait envie de passer la parole et que nous ayons affaire à une interpellation, il faut que ce locuteur n'ait pas reçu auparavant un signe de candidature de la part de l'interlocuteur. Dans ce cas, l'interpellation correspond à une intention de céder la parole par épuisement de cette dernière ou nécessité d'obtenir un écho à cette 
parole. Le locuteur n'ayant plus rien à ajouter à son intervention ou nécessitant une réaction de son interlocuteur, la sollicite en l'interpellant pour qu'il prenne à son tour la parole. Cette demande pourrait prendre la forme de :

-Regards plus ou moins prolongés pour annoncer la fin de l'intervention et solliciter la réaction de l'autre avec accompagnement éventuel d'une mimique d'attente.

-Pause plus ou moins longue marquant l'attente d'une réaction de la part de l'interlocuteur.

-Un syntagme conclusif du type : « voyez-vous... », « et voila... »...

-Un questionnement verbal directement adressé à l'allocutaire du type : "Alors ?», «Qu'en pensez-vous? »...

-Une montée sonore finale avec maintien de la sonorité finale comme un point de suspension appelant à une réaction.

-Un geste déictique en direction de l'allocutaire désigné.

Un mouvement de retrait ou détournement du regard (cf. photo ci-après)

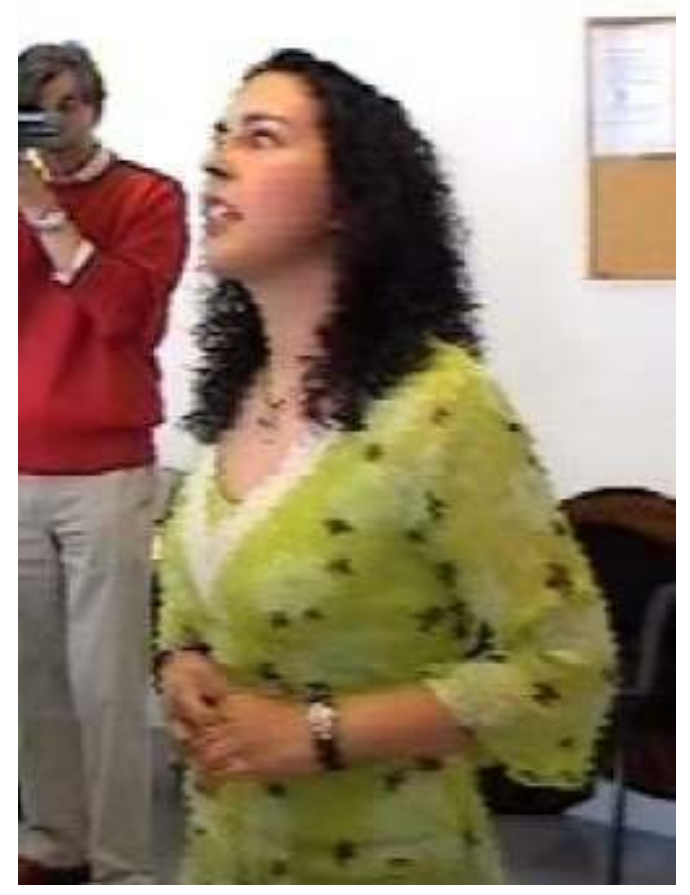

17 Si l'interlocuteur fait acte de candidature à la parole, c'est qu'il n'a pas reçu auparavant de sollicitude de la part du locuteur et qu'il nécessite de s'interposer dans le discours développé par le locuteur. Dans le premier cas, il ne ferait que réagir positivement ou négativement à la sollicitation en question. La candidature plus ou moins spontanée à la parole de la part de l'interlocuteur pourrait prendre la forme de :

-Gestes d'impatience (mouvements nerveux, changements de posture, détournements de regards, mimiques de tension...).

-Regard prolongé et insistant, avec mimique et éventuellement les sourcils vers le haut, illustrant une requête de la parole.

-Raclements de gorge plus ou moins sonores.

-Mouvements du tronc vers l'avant comme si l'interlocuteur allait s'élancer pour prendre la parole.

-Inspirations plus ou moins profondes et sonores comme pour prendre la parole avec mouvements des lèvres vers l'avant. 
-Onomatopées interjectives avec intonations d'interruption : « mais ! », «Et... ! »...

-Faux-départs verbaux avec le « je » très présent.

-Gestuelle des bras et/ou des mains comme pour interrompre le locuteur (cf. photo ciaprès).

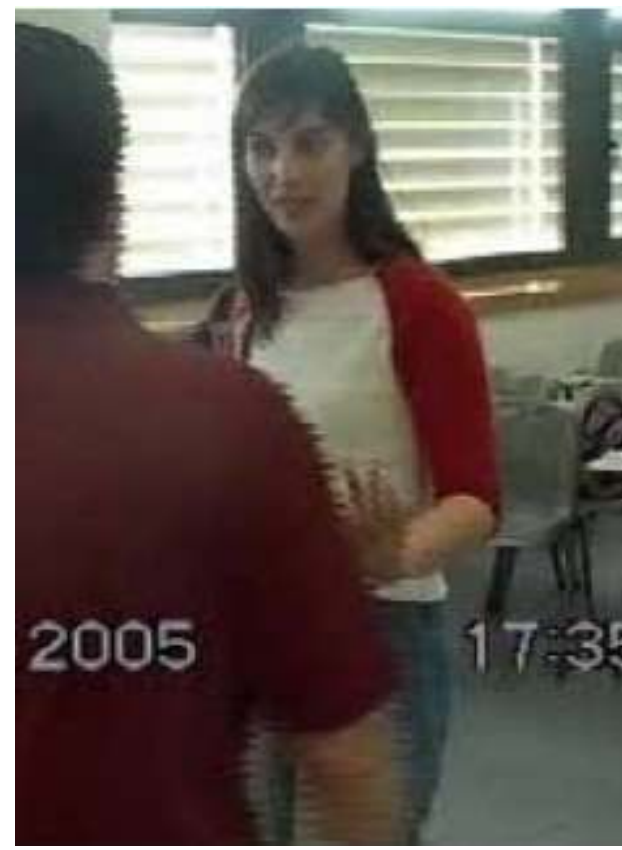

19 Une fois dégagés les indices possibles d'interpellation comme signes de gestion de la parole dans les interactions orales, de face à face ou autres, il nous faut analyser les interpellations en tant que commentaires pour ce qui est de l'évaluation cognitive ou empathique qui se dégage de la relation, le tout prenant la forme de marqueurs pouvant être aussi bien kinésiques, vocaux et/ou verbaux.

\subsection{Analyse de l'interpellation comme marqueur d'animation dans les interactions orales}

Le marquage d'animation du discours dans l'interaction orale, de face à face ou autre, peut être de deux grands styles différents, en l'occurrence celui qui permet aux participants à l'interaction d'évaluer leur compréhension réciproque et celui qui permet aux interlocuteurs de commenter le discours propre à l'interaction en l'évaluant de manière positive ou négative. Il s'agit de deux types de signes bien distincts dans leur fonction et, comme nous l'avons signalé auparavant, ayant en parallèle un rôle systématique de signe de gestion de la parole. Il n'est pas toujours aisé de déterminer la fonction animatrice et évaluatrice des signes. Toutefois, en suivant l'évolution sémiotique de l'interaction et en se concentrant sur les réactions aux signes observés, la nature même de ces réactions permet d'identifier si nous avons affaire à un signe ayant une valeur de marquage de l'évaluation cognitive ou de commentaire d'évaluation empathique. Or, cette analyse plus profonde, plus précise, permet de mieux comprendre pourquoi une interpellation en tant que passation ou candidature atteint son objectif ou pas, en l'occurrence celui de modifier le cours des événements ou le flux de l'interaction en cours. Au-delà de la différence de signes dans les deux cas, pour ce qui est de l'évaluation cognitive, le locuteur interpelle son interlocuteur pour lui passer la parole, ou réciproquement, et attend en même temps un type de réaction concrète quant à la 
compréhension de ses propos de la part de l'interlocuteur et, sans doute, avec l'espoir de reprendre la parole plus rapidement que dans le premier cas, dans la mesure où la réaction de l'interlocuteur, positive ou négative, a de fortes chances de lui renvoyer cette parole pour l'inviter à poursuivre ou pour lui demander des explications ou précisions supplémentaires. Nous voyons donc comment toute interpellation a une valeur de gestion de la parole, mais au-delà possède aussi une valeur de marqueur d'animation, soit pour marquer une évaluation cognitive, soit pour commenter de manière empathique l'interaction en cours et cette dernière fonction nous éclaire plus précisément sur les intentions des interlocuteurs tout en pouvant nous renseigner également quant à l'évolution de l'improvisation de l'interlocution

\subsubsection{Analyse de l'interpellation comme marqueur d'évaluation cognitive dans les interactions orales}

21 L'interpellation comme évaluation cognitive peut intervenir au cours de tout le processus évolutif de cette interaction. En respectant le concept d'interpellation tel que nous l'avons défini au début du texte, nous aurions deux grands types d'interpellation ayant la fonction de marqueur d'évaluation cognitive, en l'occurrence des signes servant au locuteur pour évaluer la compréhension de son ou ses interlocuteur(s) d'un côté (avec le locuteur passant la parole à l'interlocuteur, donc une valeur simultanée de gestion de la parole) et, de l'autre, des signes servant à l'interlocuteur afin d'exprimer son incompréhension au locuteur (marqueur ayant simultanément une valeur de candidature à la parole). Il s'agit en effet des deux seuls types de marqueurs d'évaluation cognitive qui, en tant qu'interpellations d'autrui, sont susceptibles d'interrompre le flux du discours pour une vérification de la compréhension de l'allocutaire d'une part et, d'autre part, pour manifester clairement une incompréhension de la part de cet allocutaire avant que le locuteur poursuive son discours. Dans le premier cas, l'évaluation cognitive du locuteur ne peut faire suite à une évaluation négative de l'interlocuteur. En effet, il faut que le marquage soit spontané et l'interpellation s'appuie sur la nécessité de vérification de la compréhension avant d'aller plus loin dans le discours. Dans le second cas, le marquage d'interpellation ne peut pas non plus faire suite à une interpellation du locuteur pour vérifier la compréhension du discours. Il s'agit d'une interpellation plus ou moins spontanée de la part de l'interlocuteur. Nous voyons ainsi comment dans les deux cas le caractère spontané de la désignation et de l'interposition se révèle fondamental.

Pour ce qui est des marqueurs d'interpellation servant à vérifier la compréhension de l'allocutaire de la part du locuteur, ils interviennent à des moments précis de l'interaction lorsque le locuteur sent la nécessité de vérifier que son interlocuteur n'ait pas de problème de compréhension. En fait, cette interpellation illustrée par un marqueur d'évaluation cognitive résulte d'une nécessité impérieuse du locuteur de s'assurer que son interlocuteur le suit sans problème, en général suite à un discours long ou complexe ou incertain dans sa formulation, comme dans le cas des interactions orales, de face à face ou autres, dans une langue étrangère peu ou pas maîtrisée. L'interpellation évaluant spontanément la compréhension de l'interlocuteur pourrait prendre la forme de :

-Interpellations verbales courtes et interrogatives: «D'accord ?", «OK ?», «Oui ?», «Hein?»...

-Interpellations verbales plus longues et explicites : « Tu comprends, oui ou non?»... 
-Montées sonores et suspensives doublées éventuellement d'un regard en direction de l'interlocuteur

-Regards prolongés avec éventuellement haussement de sourcils et silence afin de vérifier la compréhension de l'interlocuteur (cf. photo de droite ci-après)

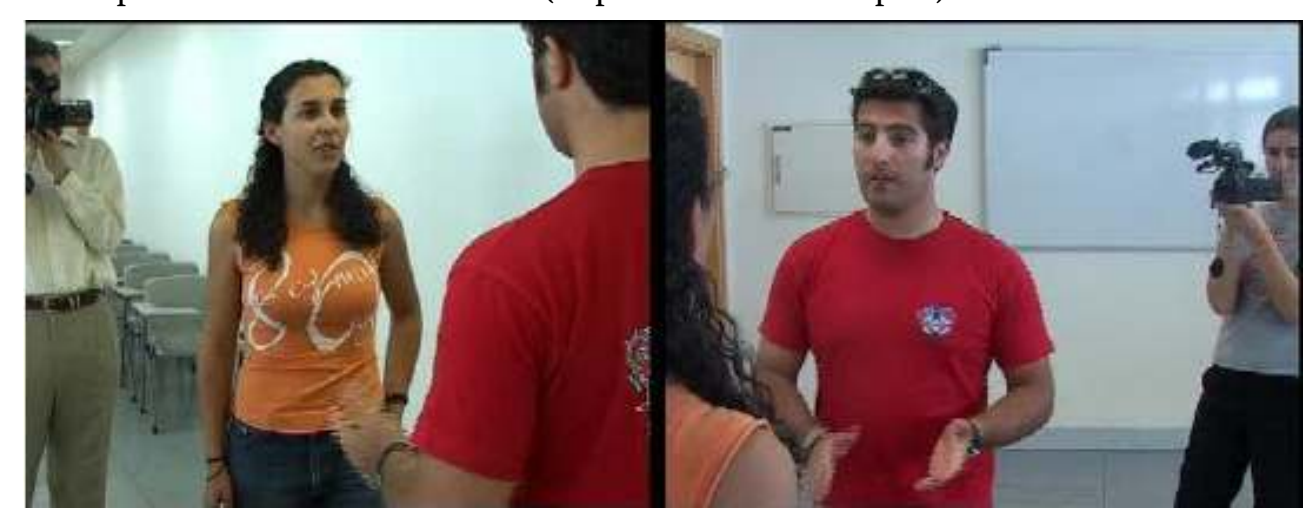

Nous voyons sur la photo de droite comment le locuteur interrompt subitement son discours pour interroger du regard son allocutaire afin de savoir si elle suit ce qu'il est en train d'expliquer. Grâce à la deuxième caméra, nous voyons comment l'interlocutrice attentive n'émet simultanément aucun signe d'incompréhension, ce qui nous permet d'envisager l'existence d'une interpellation ayant un caractère d'évaluation cognitive de la part du locuteur en interaction.

Pour ce qui est des marqueurs d'interpellation servant à exprimer l'incompréhension au locuteur de la part de l'allocutaire, ils interviennent lorsque ce dernier perd le fil du discours du locuteur. Il s'agit d'une interpellation très spontanée, mais pas nécessairement immédiate. De fait, l'interlocuteur peut attendre quelques instants l'évolution du discours avant d'interpeller le locuteur, mais pas trop dans la mesure où le locuteur devra percevoir ce que son interlocuteur n'a pas compris. Dans le cas des interactions orales, de face à face ou autres, dans une langue étrangère peu ou pas maîtrisée, de telles interpellations sont très fréquentes et elles peuvent prendre la forme de :

-Emissions brèves interjectives d'incompréhension : « Quoi ? », « Hein ? »...

-Interpellations plus longues d'incompréhension: «Excuse-moi, mais je ne comprends pas!»...

-Gestuelles d'interruption/interpellation pour demander des précisions

-Mouvements de tête latéraux accompagnés éventuellement d'un froncement de sourcils et/ou d'une mimique d'incompréhension

-Mimiques d'incompréhension avec sourcils froncés et moue buccale (cf. photo de droite ci-après) 


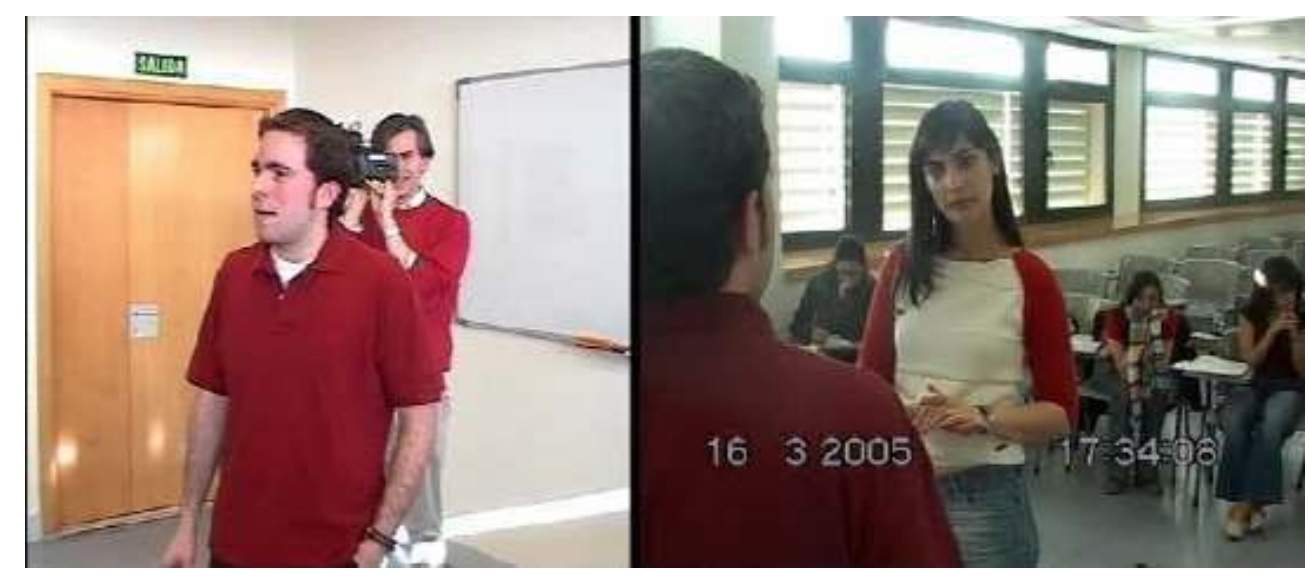

\subsubsection{Analyse de l'interpellation comme marqueur de commentaire empathique dans les interactions orales}

Comme pour les marqueurs d'évaluation cognitive, ceux exprimant un commentaire empathique, ou une évaluation affective afin d'animer l'interaction en cours, ont aussi une fonction de gestion de la parole, soit pour la passer à l'interlocuteur dans le cas du locuteur, soit pour être candidat à cette parole dans le cas de l'interlocuteur. Il s'agit donc d'interpellations empathiques, à valeur positive ou négative, de la part du locuteur pour solliciter la réaction de l'interlocuteur en lui proposant de prendre la parole, ou d'interpellations empathiques, à valeur positive ou négative, de la part de l'interlocuteur afin de réagir au discours du locuteur tout en sollicitant la parole. Dans le premier cas, le locuteur anime l'interlocuteur à réagir, donc à prendre la parole, au moyen de marqueurs conatifs (verbaux, kinésiques ou acoustiques) ou de marqueurs expressifs essayant d'influencer cet interlocuteur de manière positive ou négative. Dans le second cas, nous retrouvons les mêmes marqueurs pour l'interlocuteur avec l'objectif d'influencer positivement ou négativement le discours du locuteur par le biais d'une prise de parole affirmant la candidature. C'est ainsi que dans le cas d'un locuteur exprimant une évaluation empathique, les marqueurs de commentaire pourraient prendre la forme de : -Interpellations verbales interjectives pour influencer autrui : « Silence ! », « Génial ! »...

-Interpellations d'injures verbales, kinésiques et/ou sonores exprimant des sentiments malveillants vis-à-vis de l'autre...

-Mimiques faciales, mouvements corporels, exclamations interjectives expressives de joie, de surprise, de peur, de colère, de dégoût, de tristesse ...

-Interpellations kinésiques et/ou vocales pour influencer autrui : Index placé devant les lèvres, mouvements de bras et de mains illustrant une énorme satisfaction, regard admiratif porté sur soi ou sur l'autre, exclamations sonores diverses (cf. photo ci-après) 


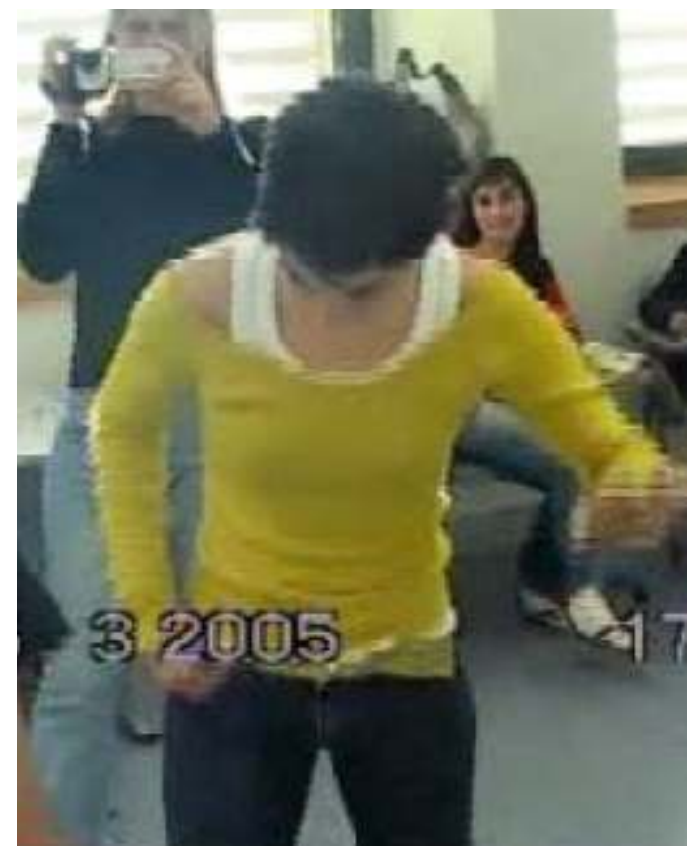 Interpellations d'injures verbales, kinésiques et/ou sonores exprimant des sentiments malveillants vis-à-vis de l'autre...

Mimiques faciales, mouvements corporels, exclamations interjectives expressives de surprise, de peur, de colère, de dégoût, de tristesse, de joie (cf. photo de gauche ci-après)

37 Nous voyons sur la photo de gauche, grâce aux deux caméras filmant simultanément, comment l'allocutaire réagit par un long sourire aux explications données par le locuteur et comment ce commentaire empathique est spontané car en rien provoqué par le discours de ce dernier vu que le locuteur en question est totalement concentré sur son explication.

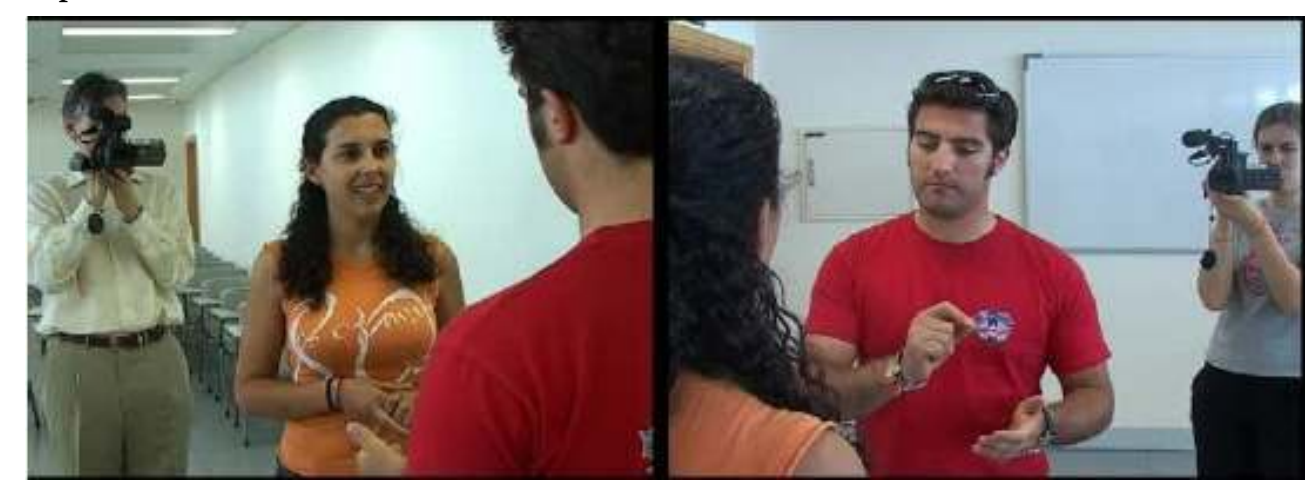




\subsection{Analyse de l'interpellation comme marqueur d'appel à l'aide dans les interactions orales}

Il s'agit d'une hypothèse d'interpellation qui ne serait pas aussi explicite que les autres puisque ne s'adressant pas directement à l'interlocuteur. Pourtant, il semble que certains signes que nous appelons extra - communicatifs ont une charge potentielle d'interpellation de l'autre qui serait en quelque sorte plus inconsciente que consciente puisque c'est le comportement même de l'un des interlocuteurs qui pourrait interpeller le ou les interlocuteurs. Nous aurions affaire alors à des signes extra - communicatifs illustrant une difficulté à assumer l'interaction, de face à face ou autre, révélant une anxiété face à l'activité en cours ou encore une crainte de ne pas être à la hauteur des attentes d'autrui par rapport à cette activité. De tels signes sont très fréquents, surtout dans les interactions orales de face à face, et sont facilement observables de par leur caractère incontrôlé et le fait qu'ils attirent rapidement l'attention des interlocuteurs. Plus que l'expression d'une fuite, le signe extra - communicatif révèle une difficulté et suggère un appel à l'aide ou pour le moins à la compréhension de l'autre dans l'interaction. Une telle dimension me permet de penser que nous sommes face à un type d'interpellation implicite de l'autre dans l'interaction orale prioritairement de face à face, interpellations que nous pourrions nommer indirectes, car permettant à leurs acteurs de solliciter une parenthèse dans l'interaction à travers un mouvement de soulagement qui peut prendre la forme de :

-Marqueurs self-adaptateurs de mouvements de confort: croisements de jambes, croisements de bras, changements de positions, bâillements ...

-Marqueurs adaptateurs aux objets: manipulation d'objets (jouer avec un stylo, un crayon, égrener un boulier...), activités ludiques (dessiner automatiquement, plier un papier ...)

-Marqueurs self-adaptateurs autocentrés : Marqueurs self-adaptateurs autocentrés : rires nerveux, éclats de rires, gestes autocentrés (grattages, tapotements, onychophagie, balancements, stéréotypies motrices...cf. photo de gauche ci-après)

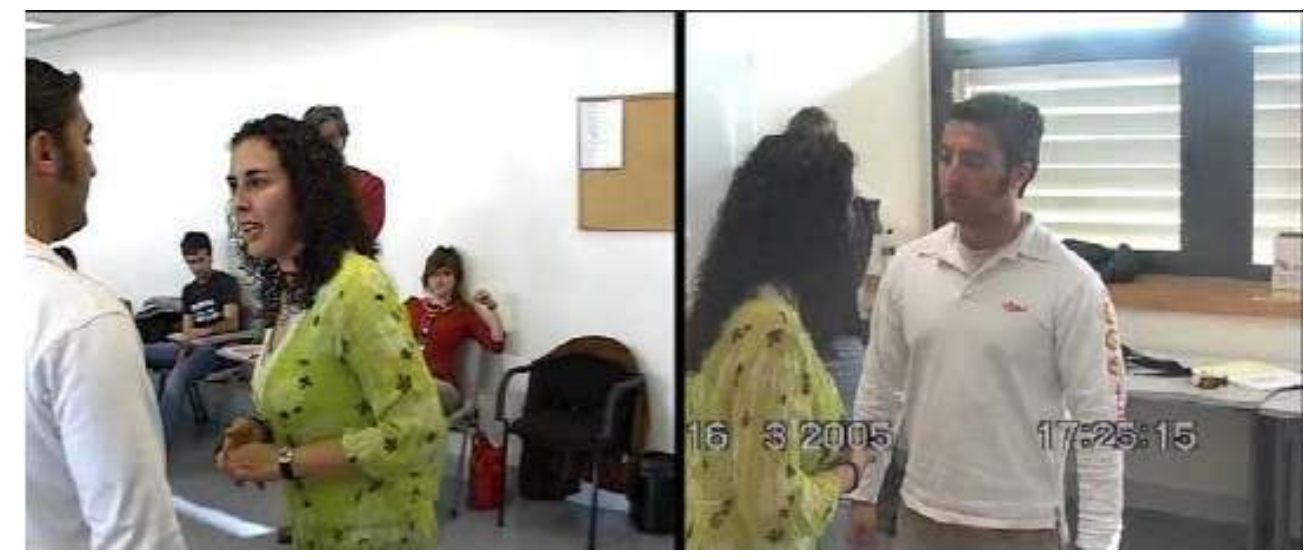

Sur la photo de gauche, nous voyons comment la locutrice s'appuie sur un auto-contact à travers un frottement de mains afin de surmonter un moment un peu difficile de l'interaction orale de face à face, ce moment pouvant être provoqué éventuellement par l'attitude de son allocutaire. Ce genre de signes est très fréquent dans les interactions, a fortiori si elle se développe dans une langue étrangère peu ou pas maîtrisée. 


\section{Comment utiliser méthodologiquement les indices d'interpellation dans les classes de FLE ?}

Interagir ou dialoguer oralement avec autrui suppose une compétence de communication que tout le monde ou presque s'accorde à voir comme la compétence majeure intégrant toutes les autres. De fait, il est possible d'établir une liste de savoirs, de savoir-être, de savoir-devenir et de savoir-faire propres à l'interaction orale, ces savoirs, ces savoir-être, ces savoir-devenir et ces savoir-faire illustrant des compétences certes linguistiques, mais également sociolinguistiques et pragmatiques. Dans le premier cas, les participants à l'interaction orale nécessitent du lexique et de la grammaire en relation avec le discours partagé, mais aussi des connaissances et des savoir-faire phonologiques ainsi que des compétences sémantiques afin de contrôler l'intercompréhension. Pour ce qui est des compétences sociolinguistiques, les apprenants doivent faire appel à un ensemble de marqueurs de la relation sociale dont les signes d'interpellation qui nous intéressent (CECR, 2000, 93). Enfin, au niveau des compétences pragmatiques, les apprenants s'appuient sur des compétences discursives basées sur des ressources stratégiques plus ou moins élaborées selon les niveaux langagiers et qui traitent entre autres la souplesse, les tours de parole, la cohérence et la cohésion. (CECR, 2000, 96-97) A ces compétences, il faut ajouter des compétences fonctionnelles à visées plus particulières. Or, toutes ces compétences se basent sur des savoirs, des savoir-être, des savoir-devenir et des savoirfaire en termes de ressources de la continuité (Brouté, 2008) et peuvent être évaluées avec un certain degré de précision. D'autre part, tous ces savoirs, savoir-être, savoir-devenir et savoir-faire peuvent se traduire par un ensemble de tâches et d'activités à réaliser avec des objectifs à atteindre démontrant la pertinence d'une compétence dialogique ou d'interaction orale que nous allons approfondir.

Au-delà même du fait que nous avons affaire à l'activité principale développée dans toute classe de Langue Etrangère ou autre matière et à l'un des supports les plus pertinents pour la construction du langage et des connaissances, la compétence d'interaction orale relève d'un particularisme. En effet, les compétences en jeu dans l'interaction orale sont tributaires des aspects particuliers de cette activité. C'est ainsi que l'interaction orale se différencie de toutes les activités développées lors d'un enseignement/apprentissage de Langue Etrangère dans la mesure où elle est la seule qui ne peut se réaliser individuellement. Le fait qu'il y ait toujours au moins deux participants à l'activité doit donc être pris en considération pour l'appréciation des compétences en jeu et, par conséquent, leur évaluation. De plus, il ne faut pas oublier que les participants changent nécessairement de rôle au cours de l'activité, passant alternativement de celui de locuteur à celui d'allocutaire, chaque rôle supposant ses propres habiletés et, par conséquent, des compétences différentes.

Par ailleurs, être compétent en production ou expression orale en langue étrangère suppose la mobilisation de ressources et leur adéquation à la tâche requise. Les tâches en question peuvent être de l'ordre d'un exposé, de la lecture d'un texte à haute voix ou d'une annonce publique par exemple. Dans ce cas, toutes les ressources langagières de compensation et de reformulation proposées par le CECR $(2000,53-54)$ correspondent aussi à la compétence dialogique du locuteur dans les interactions orales, de face à face ou autres. C'est ainsi que, «en l'absence de ressources suffisantes, l'utilisateur/apprenant peut être conduit à modifier ce qu'il/elle voulait effectivement dire pour s'en tenir aux 
moyens linguistiques disponibles. » (CECR, 2000, 53) Mais, de même, il/elle peut tenter d'améliorer son discours en développant stratégiquement des reformulations paraphrastiques ou pas (Brouté, 2007, 2008) Par ailleurs, toutes les autres compétences linguistiques, sociolinguistiques et pragmatiques qui entrent en jeu dans l'expression orale sont pertinentes pour l'interaction orale. Quant à la réception ou compréhension orale, les compétences à développer en Langue Etrangère supposent des ressources permettant de construire un sens à partir d'opérations de cadrage, d'identification d'indices linguistiques, sociolinguistiques et pragmatiques, et l'élaboration d'hypothèses sur le sens perçu et les intentions communicatives sous-jacentes, ces hypothèses pouvant être révisées par la suite. Toutes les activités de réception/compréhension orale requièrent ces compétences qui sont aussi sollicitées de manière pertinente dans les interactions orales, de face à face ou autres.

Cependant, toutes les habiletés de production et/ou de réception orales entrent certes en jeu dans les différentes interactions orales, mais d'une manière propre à l'interactionnel, ce qui suppose une adaptation des ressources évoquées, et notamment celles liées aux interpellations, au caractère alternatif et presque simultané des interactions d'une part et, d'autre part, à la prise en compte de compétences liées à la relation des êtres communiquant oralement en face à face ou d'une autre façon.

\subsection{Des activités préparatoires pour mettre en valeur l'importance des différentes interpellations pour le développement d'interactions orales, de face à face ou autres, en FLE}

Malgré l'usage fréquent des interpellations dans la langue maternelle ou de référence, les élèves qui étudient une langue étrangère ont tendance à geler leurs capacités à les utiliser lorsqu'ils se retrouvent sur le terrain d'enseignement/apprentissage. Aussi, il faut développer un ensemble d'activités réflexives et pratiques qui leur permettent d'assurer une continuité entre leur pratique quotidienne des interactions orales et de leurs interpellations, et leurs pratiques de classe à travers la culture et la langue étrangères.

\subsubsection{Des ateliers de réflexion conceptuelle}

Il s'agit d'un ensemble d'activités interactionnelles de groupes (entre 3 et 5 élèves) à travers lesquelles les élèves réfléchissent ou sur un concept comme celui de l'interpellation en cherchant à le définir le plus précisément possible ou sur leur propre pratique d'interactions orales en leur demandant de réfléchir sur leurs façons habituelles d'aborder ces interactions orales ou de donner la parole à l'autre ou de la demander lorsque l'autre parle. Ces réflexions sont mises en commun à la fin de l'atelier au niveau du groupe/classe.

Exemples :

-Le défi de la définition (Brouté, 2008, p. 24)

Ateliers de réflexion sur une pratique : Comment aborder une personne afin d'avoir une conversation avec elle? Comment faites-vous pour signaler à votre interlocuteur que vous lui passez la parole en interaction?... 


\subsubsection{Des activités interactionnelles pour fomenter l'usage des différents types d' interpellations}

\section{d'intervention (appellatifs, nominatifs ...) et de désignation}

-Les situations problématiques (Brouté, 2008, pp. 35-36) avec notamment la reconstruction de puzzles à distance

-La statue à reconstruire à distance (Brouté, 2008, p. 12) pour travailler les interpellations d'intervention et de désignation (cf. photo ci-après où le locuteur interpelle les autres à distance pour créer une statue)

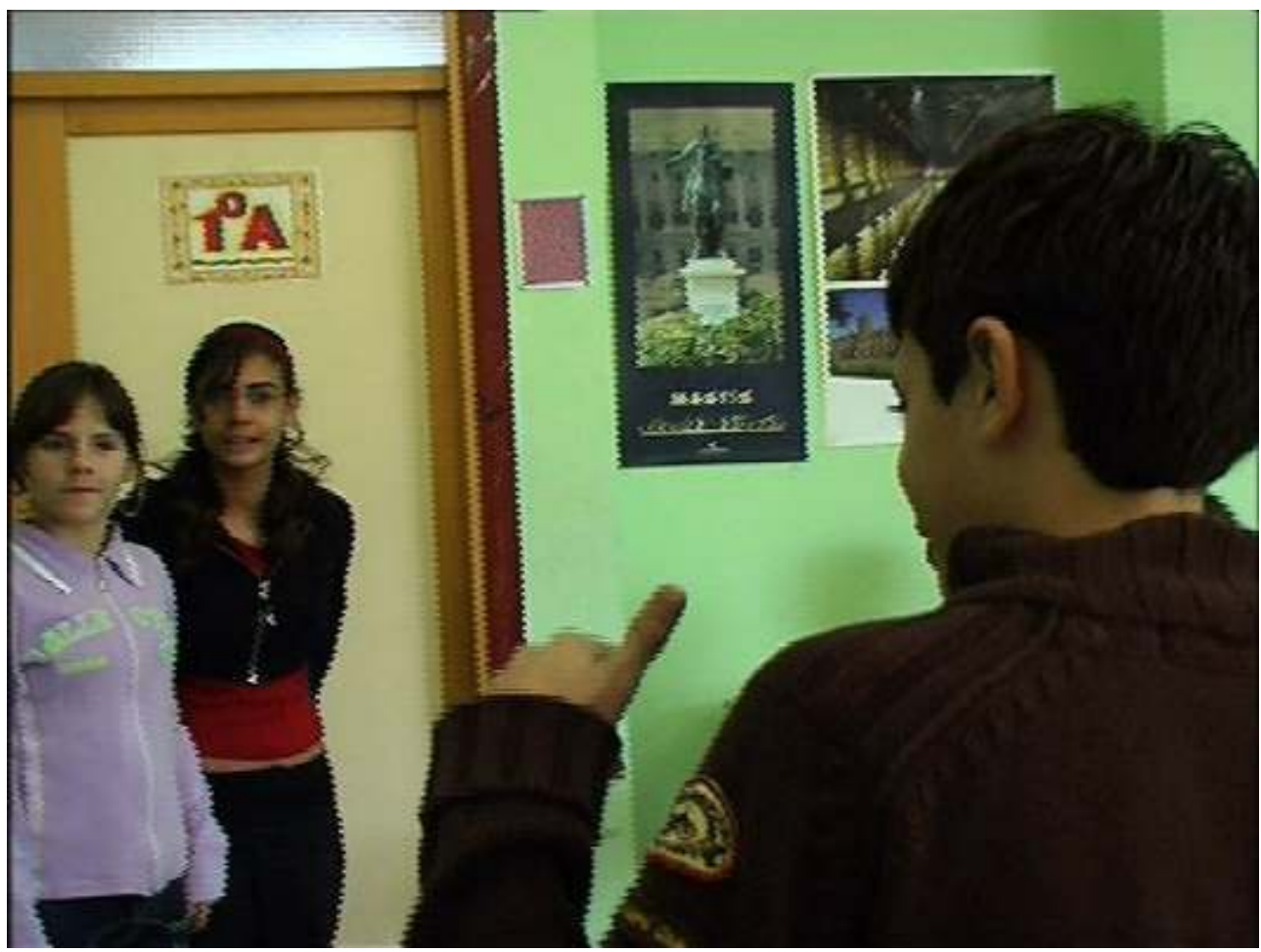
consciemment des interpellations

\subsection{Des activités interactionnelles pour suivre l'évolution de l'utilisation des indices d'interpellation}

Presque toutes les activités interactionnelles et les jeux de rôles peuvent entrer dans cette catégorie bien que certaines sont plus propices que d'autres au développement et au 
suivi des interpellations. En fait, à ce stade du travail didactique sur l'interpellation, il est important d'observer le plus précisément possible l'utilisation des interpellations dans les activités interactionnelles et les jeux de rôles qu'ils soient transformés ou pas. A ce titre, l'usage le plus fréquent possible d'un moyen d'enregistrement vidéo est recommandable. Voyons certains types d'activités plus propices à un tel suivi et son évaluation.

\subsubsection{Des activités interactionnelles non transformées avec suivi évaluatif des indices d'interpellation utilisés plus ou moins inconsciemment} les canaux prosodiques et verbaux)

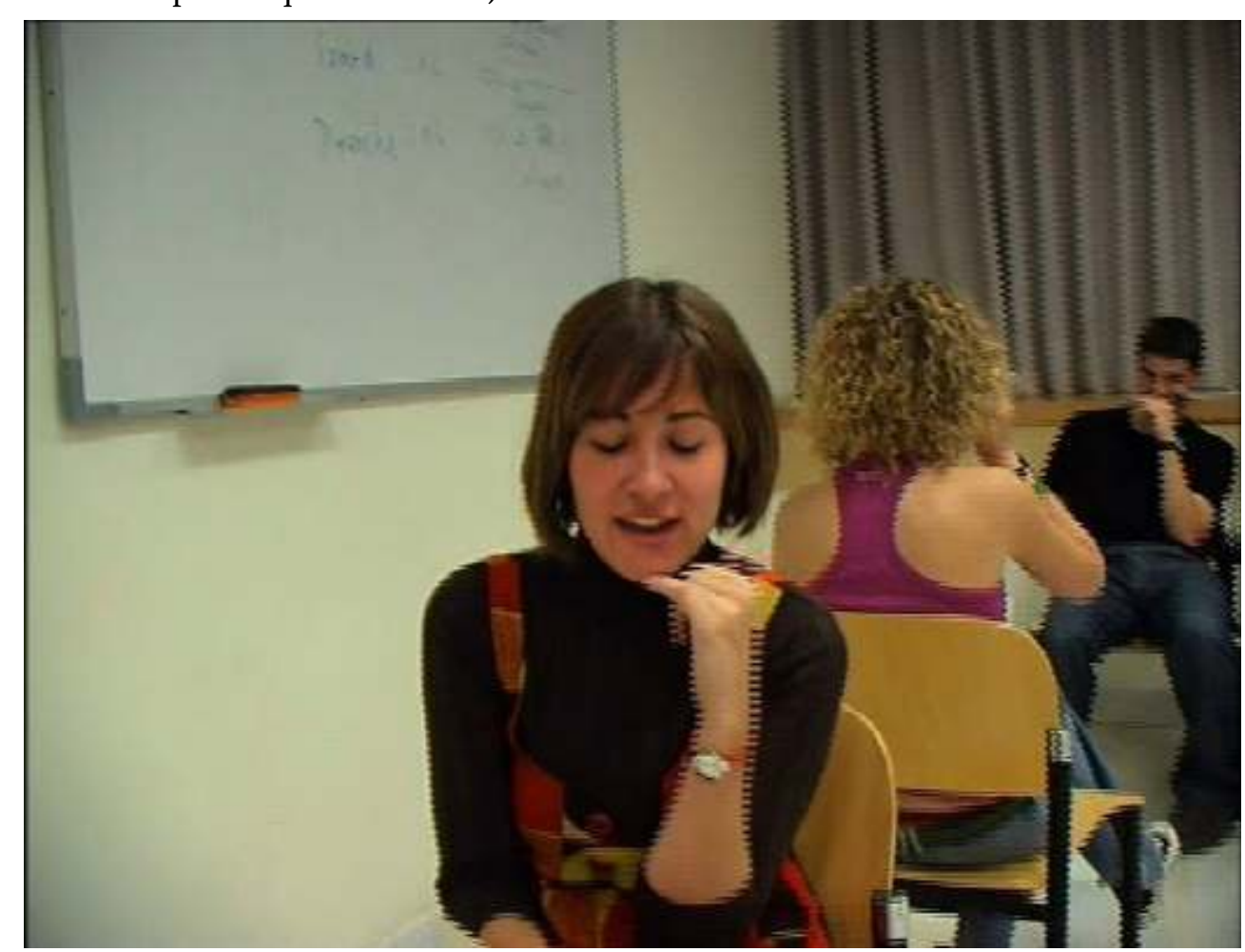

\subsection{Des activités interactionnelles pour remédier à un manque d'utilisation des indices d'interpellation}

Une fois observées et analysées les interactions orales transformées ou pas, certaines activités de remédiation peuvent permettre d'aider les élèves à pallier progressivement 
leur manque d'utilisation d'interpellations dans leurs productions et leurs discours. Voyons certaines activités susceptibles de favoriser l'objectif précité.

\subsubsection{Des activités de remise à jour des pratiques en matière d'interpellations dans les interactions}

-Atelier de réflexion approfondie sur les modes d'utilisation des interpellations lors des interactions orales dans la vie quotidienne par rapport aux manques mis en avant

\subsubsection{Des activités interactionnelles de remédiation au manque d'utilisation des interpellations}

-L'histoire en chœur (Brouté, 2008, p. 121) pour la remédiation kinésique de l'interpellation par le regard

-Sujets aimés, sujets haïs (Brouté, 2008, p. 124) où la prise de parole et sa passation sont vitales par le biais de l'interpellation verbale

-Le guide, l'aveugle et le trésor (Brouté, 2008, p. 117) pour la remédiation prosodique de l'interpellation (cf. photo ci-après où l'on peut voir une apprenante qui ne peut bouger qui guide acoustiquement et verbalement à un autre apprenant vers un objet requis)

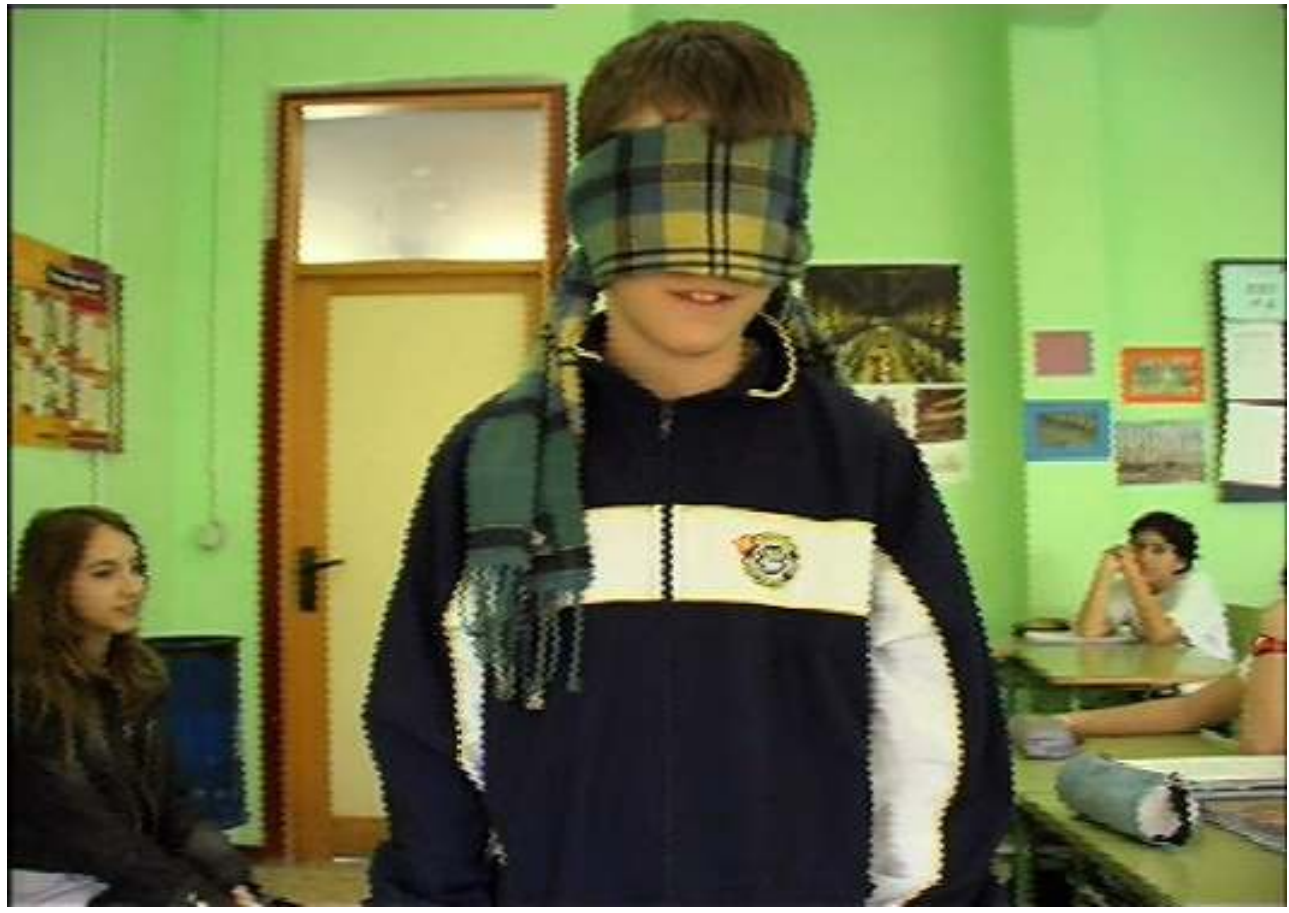

\section{BIBLIOGRAPHIE}

Académie Française (1932-1935) : Dictionnaire de l'Académie Française, Hachette, Paris (8ème édition) 
Brouté A. (2008): Les interactions orales dans la classe de F.L.E., Pearson Educación, Madrid, 128 pages (accompagné du DVD « C'est parti ! » de 1h17).

Brouté A. (2007): De nouveaux descripteurs pour évaluer les interactions orales en langue étrangère. Revue D. I. L. L. (Didáctica, Lengua y Literatura), Madrid, 29 pages.

Brouté A. (2006): Transformation, parole et ressources de la continuité: méthodologie de l'interaction orale dans l'apprentissage d'une langue étrangère. ANRT Diffusion, Université de Lille, 573 pages.

Conseil de la compétence culturelle (2000) : Un Cadre Européen Commun de Référence pour les langues : apprendre, enseigner, évaluer. Editions Didier.

De Raymond J-F. (1980) : L'improvisation, Librairie philosophique, J. Vrin, Paris, 231 pages.

Duncan S., Fiske D.W. (1977): Face to face Interaction Research, Methods and Theory, Hillsdale, NJ: Laurence Erlbaum.

Goffman E. (1991): Les cadres de l'expérience. Editions de Minuit. Paris. 573 pages. Traduction de Frame Analysis (Isaac Joseph; Ed. Originale, 1974, Harper \& Row, New York).

Goffman E. (1973, a) : La mise en scène de la vie quotidienne : La présentation de soi, Editions de Minuit, Paris.

Goffman E. $(1973$, b) : La mise en scène de la vie quotidienne : Les relations en public, Editions de Minuit, Paris.

Gülich E., Kotschi T. (1995): Discourse Production in Oral Communication, in Quasthoff U. M., Aspects of oral Communication, Berlin, New York, Walter de Gruyter. (Pages 30-66)

Le Robert \& CLE international (1999): Dictionnaire du français, sous la direction de Josette Rey Debove, Paris, 1232 pages.

Sacks H., Schegloff E., Jefferson G. (1974): A Simplest Systematic For the Organization of TurnTaking in Conversation in Language 50. (Pages 696-735)

\section{RÉSUMÉS}

Dans cet article, je présente une analyse et des exemples concrets de ce que peut représenter l'interpellation dans des interactions orales, de face à face ou autres, transformées et développées par des apprenants de Français Langue Etrangère (F.L.E.), l'interpellation étant vue comme une demande d'interlocution ou signe manifeste de la participation et de la coopération dans un groupe. Je commenterai des exemples d'indices d'interpellation tant verbaux qu'acoustiques et/ou kinésiques afin de montrer combien l'observation, la description et l'utilisation de ces indices peut être déterminante à l'heure de promouvoir des activités motivantes ou remédier à des difficultés d'expression dans le cadre d'un enseignement / apprentissage de la compétence en Interaction orale de face à face en langue étrangère et/ou en langue maternelle (Brouté, 2007). Je présente aussi une analyse et une interprétation de certains signes extra-communicatifs afin de souligner leur rôle particulier en tant qu'interpellations du devenir de l'interlocution ou signes d'appels à l'aide dans la recherche des ressources adaptées afin de sauver la face. Enfin, je suggèrerai quelques éléments de méthodologie afin d'utiliser les indices précités comme ressources de développement de la compétence d'Interaction orale en Langue Etrangère, cette dernière activité pouvant être parfois l'objectif principal de l'enseignement/apprentissage en question et représentant presque toujours le moyen le plus 
puissant de développement de l'apprentissage en question grâce à l'usage de la parole dans le contexte de la classe que ce soit de manière expressive ou interactionnelle.

In this article, I present an analysis and concrete examples of the fact that can represent the interpellation in oral interactions transformed and developed by students learning French like Foreign language (F.L.E), the interpellation being seen as a demand of interlocution or sign shows of the participation and some cooperation in a group. I shall comment on examples of indications of interpellation so much verbal as acoustic and \or kinesics to show how much the observation, the description and the use of these indications can be determining to promote rewarding activities or remedy difficulties of expression within the framework of an education / learning of the competence in oral Interaction in foreign language and \or in motherly language (Brouté, 2007). I also present an analysis and an interpretation of certain extra-communicative signs to underline their particular role as interpellations of the future of the interlocution or the signs of calls for help in the research for the resources adapted to save face. Finally, I shall propose some elements of methodology to use the aforesaid indications as resources of development of the competence of oral Interaction in foreign languages, this last activity which can be sometimes the main objective of the education / learning in question and representing almost always the most powerful means of development of the learning in question thanks to the usage of the word in the context of the class whether it is in a meaning way or in interaction.

INDEX

Mots-clés : interaction orale, interpellation, marqueurs de la relation, dynamique intentionnelle

\section{AUTEUR}

ALAIN BROUTÉ

Université Autonome de Madrid (Espagne) 